\title{
Review of: "Orthogonal control of gene expression in plants using synthetic promoters and CRISPR-based transcription factors"
}

\author{
Gopal Pandi ${ }^{1}$ \\ 1 Madurai Kamaraj University
}

Potential competing interests: The author(s) declared that no potential competing interests exist.

Indeed it's nice approach to develop orthogonal control system which can work independently in plant without interfering plant system . The whole experiment is systematic, aligned different part of transcriptional unit to create one transcriptional unit subsequently aligned multiple transcriptional unit to assemble into single circuit. It can be expressed simultaneously to a specific transcription unit under it's related gRNA promoter.

However the orthogonal system can potentially work in other higher plant system, which need to be improved for the lower plant system. We were delighted to read the article.

In the infiltrated parts, it will be ideal to have more negative controls. 